\title{
Cirrhotic Cardiomyopathy in the Era of Liver Transplantation: Time for Precise Stepwise Evaluation
}

\author{
Roxana Cristina Rimbaș ${ }^{1,2}$, Mihai Rimbaș ${ }^{1,3}$, Alexandra Maria Chitroceanu ${ }^{2}$, Luiza Maria Luchian ${ }^{2}$, Corina Pop $^{1,4}$, \\ Dragoș Vinereanu ${ }^{1,2}$
}

1) Cardiology Department,

Carol Davila University of

Medicine, Bucharest;

2) Cardiology Department,

University Emergency

Hospital, Bucharest;

3) Gastroenterology

Department, Colentina

Clinical Hospital, Bucharest;

4) Gastroenterology

Department, University

Emergency Hospital,

Bucharest, Romania

\author{
Address for correspondence: \\ Corina Pop MD, PhD, \\ Associate Professor, \\ Gastroenterology Department, \\ University Emergency \\ Hospital, 169 Splaiul \\ Independenței, Bucharest, \\ Romania \\ cora.pop@gmail.com
}

\begin{abstract}
Liver cirrhosis (LC) is an important cause of mortality. Access to liver transplantation (LT) has significantly improved the prognosis of LC. A rigorous pre-transplant cardiac evaluation is mandatory, since cardiac dysfunction is considered the main cause of mortality after LT. Notwithstanding, the most updated pre-LT evaluation guidelines provide only an algorithm for the evaluation of major cardiovascular diseases, with no specific recommendations concerning cirrhotic cardiomyopathy (CCM), which is linked to various complications in LC, especially the development of heart failure after invasive procedures and surgical interventions, including LT. CCM is characterized by a cardiac dysfunction that includes systolic and/or diastolic dysfunction and/or electrophysiological abnormalities, in the absence of other known cardiac diseases. The role of the novel methods, tissue Doppler imaging and speckle tracking echocardiography, might be essential in the early detection of cardiac dysfunction, with prognosis implications in LC. All these new methods were only recently included in the CCM diagnosis algorithm. This review summarizes the old and novel techniques used for the diagnosis of CCM, with their diagnosis and prognostic role. It also highlights the strengths and the weaknesses of the new provided CCM diagnostic consensus, and proposes a step-bystep novel diagnostic algorithm, in order to better detect cardiac dysfunction.
\end{abstract}

Key words: cirrhotic cardiomyopathy - diastolic dysfunction - systolic dysfunction - cardiac biomarkers speckle tracking echocardiography - tissue Doppler imaging.

Abbreviations: ASE: American Society of Echocardiography; CCM: cirrhotic cardiomyopathy; CO: cardiac output; DD: diastolic dysfunction; EACVI: European Association of Cardiovascular Imaging; ECG: electrocardiogram; GLS: global longitudinal strain; HF: heart failure; HFpEF: HF with preserved ejection fraction; HR: heart rate; IVRT: isovolumetric relaxation times; LA: left atrium; LASr: left atrial reservoir strain; LAVi: left atrial volume; LC: liver cirrhosis; LT: liver transplantation; LV: left ventricle; LVEF: left ventricular ejection fraction; LVFP: left ventricular filling pressure; SE: stress echocardiography; STE: speckle tracking echocardiography; SVR: systemic vascular resistance; TDI: tissue Doppler imaging; TpI: troponin I; TV: tricuspid velocity; 2DE: two-dimensional echocardiography; 3DE: three-dimensional echocardiography.

\section{INTRODUCTION}

Liver cirrhosis (LC) has a

Received: 07.10.2020

Accepted: 31.10 .2020 high impact on public healthcare, representing an important cause of mortality worldwide [1]. Some patients with LC develop a progressive cardiac dysfunction, a condition named cirrhotic cardiomyopathy (CCM), that consists of an impaired ventricular performance to different stressful conditions, and may not be clinically significant at rest because of the high cardiac output (CO) and low systemic vascular resistance (SVR), both present in late stages of LC [2]. All recent data support the concept of an intrinsic myocardial dysfunction generated by various neuro-humoral substances, correlated with the severity of LC [3]. Although cardiac mortality after liver transplantation (LT) is still high, it cannot be adequately predicted by the conventional echocardiography parameters [4]. The role of novel methods, such as tissue Doppler imaging (TDI) and speckle tracking echocardiography (STE) in the diagnosis and prognosis of CCM are still a matter of debate. These innovative methods might be essential in the early detection of cardiac dysfunction in LC patients, who express 
a high variability of loading conditions. Moreover, the role of cardiac biomarkers as diagnostic criteria, and their cut-off values for the detection of cardiac dysfunction in LC patients are not yet established.

Although heart failure (HF) due to CCM is claimed to be the third cause of mortality after LT [3], the most updated pre-LT evaluation guidelines provide solely an algorithm for the evaluation of major cardiovascular diseases, and it does not give any recommendations regarding CCM $[4,5]$. Novel definition criteria were recently published in 2019, trying to incorporate significant advancements in cardiovascular imaging [6]. However, this new consensus definition has still uncovered areas that need to be improved.

This review summarizes old and novel imaging methods for the evaluation of cardiac function in LC patients, and their diagnosis and prognostic role. It also highlights the strengths and the weaknesses of the new provided CCM diagnostic criteria, and proposes a new step-by-step diagnostic algorithm for CCM that should be tested under real life conditions, and which we consider useful especially in the pre-LT evaluation. The implications of porto-pulmonary hypertension and hepato-pulmonary syndrome will not be discussed here, being a completely different entity generated by LC.

\section{DEFINITION OF CIRRHOTIC CARDIOMYOPATHY}

Cirrhotic cardiomyopathy was defined in 2005 in Montreal, Canada, as a chronic cardiac dysfunction in patients with LC, in the absence of an intrinsic cardiac disease [7]. Recently, new diagnostic criteria were provided by an expert consensus
[6]. Cirrhotic cardiomyopathy includes a variety of structural myocardial changes, systolic and diastolic dysfunction, and/ or electrophysiological abnormalities, associated with an augmented vascular function [8-10]. This cardiac dysfunction is usually asymptomatic at rest, and it is manifested as a suboptimal ventricular response at an increased demand such as LT, other major surgery, and infections [7]. Comparative diagnostic criteria for recognition of CCM, as they were provided in 2005 and 2019 consensus meetings, are described in Fig. 1, which we will refer further as "old" and "novel" criteria.

Old definition criteria were based on conventional twodimensional echocardiography (2DE), which has subsequently been proven to be able to identify only the late stages of cardiac dysfunction [8-14]. According to the last guidelines of the European Association of the Study of the Liver (EASL), conventional $2 \mathrm{DE}$ is required in all LT candidates for the preprocedural evaluation and risk stratification [4]. However, with this conventional approach, CCM often remains unrecognised. Recently, by using TDI and STE, many studies tried to better define this entity $[11,13,15-20]$. These new imaging modalities have been already validated for the detection of subclinical cardiac dysfunction in many other cardiac diseases, and TDI is now a "must" in the algorithm of diastolic dysfunction (DD) detection and left ventricular filling pressure (LVFP) characterization in all cardiac diseases [21].

Accordingly, the novel CCM consensus proposed updated criteria based on modern concepts from the HF field, including the integration of STE into routine clinical practice and the new classification of DD (Fig. 1). Similarly, serum levels of natriuretic peptides, troponin, and different profibrotic and

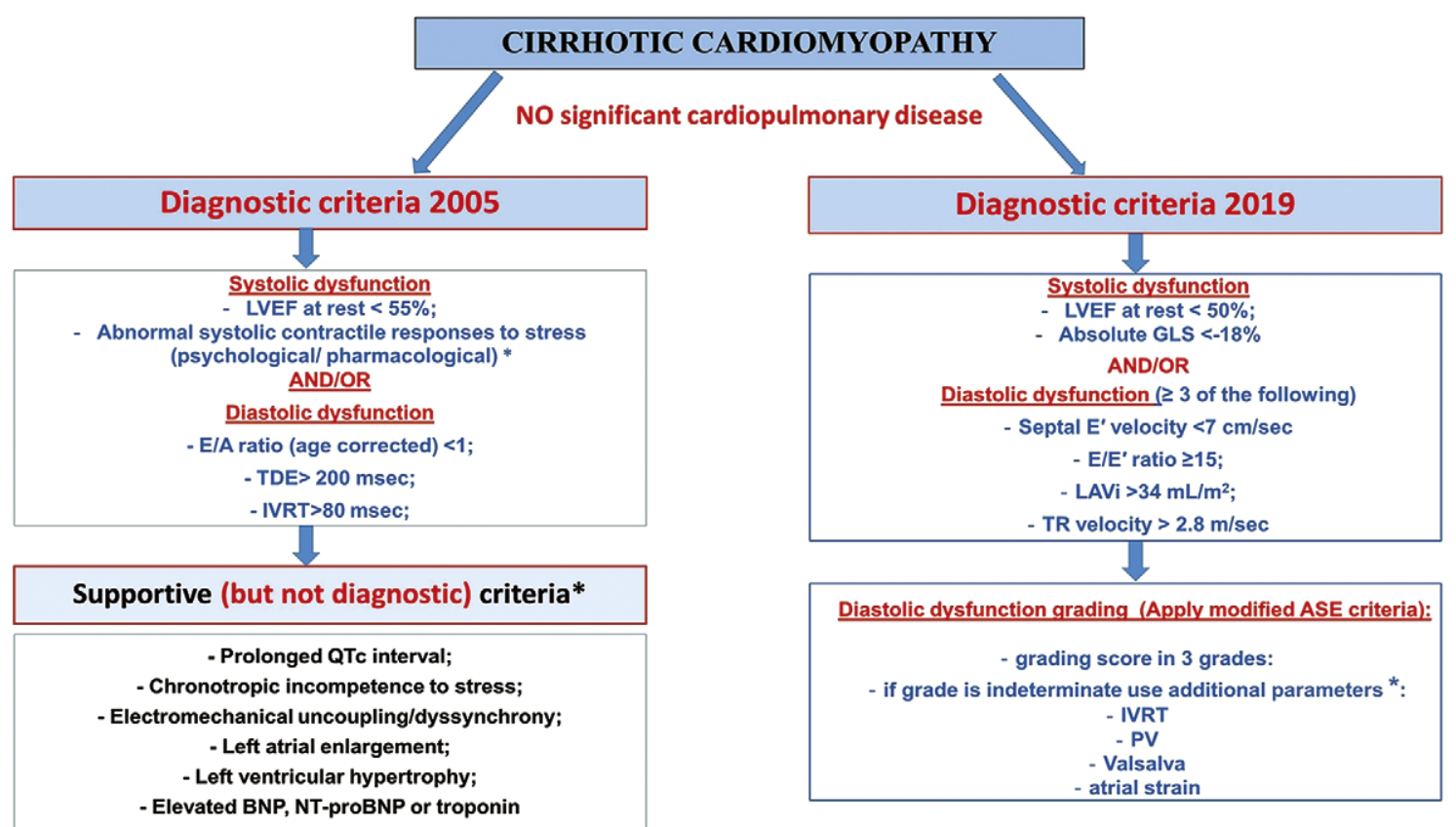

Fig. 1. Comparative diagnostic criteria of the cirrhotic cardiomyopathy according to the 2005 and 2019 definitions LVEF: left ventricular ejection fraction; E/A ratio, ratio between E, peak velocity blood flow in early diastole; A: peak velocity blood flow in late diastole; TDE: E-wave deceleration time; IVRT: isovolumetric relaxation time; QTc: corrected QT interval; BNP: brain natriuretic peptide; NTproBNP: N-terminal prohormone of BNP; GLS: global longitudinal strain; E': early diastolic velocity from the pulsed-wave tissue Doppler imaging; E/E' ratio: ratio between E and E' velocity; LAVi: indexed left atrial volume; TR: tricuspid; PV: pulmonary vein flow; ${ }^{*}$ No cut-off values/definition provided. 
proinflammatory markers are reported to be elevated in LC patients, but the role of these markers in the diagnosis and prognosis of CCM is not well established [6, 17-19].

\section{DIAGNOSTIC METHODS}

Liver cirrhosis patients should have biological, electrocardiographic, and imaging evaluation for the diagnosis of cardiac dysfunction, irrespective of the stage of LC, but especially as a part of pre-LT evaluation $[4,6]$. In early stages, DD precedes systolic dysfunction, both progressing concomitantly with the progression of the liver disease [22-24]. Since LC patients have an important peripheral vasodilation, this is a natural way of self-treating the development of overt HF $[25,26]$. Liver transplantation represents perhaps the most significant cardiac challenge in LC patients. Notwithstanding, in the perioperative period, the significant fluctuations in pre- and afterload are translated into half of these patients developing HF within the first postoperative week [23, 24]. The novel 2019 CCM definition, by including STE and an updated algorithm for DD detection, seems to make a big step forward for a better detection of cardiac dysfunction in LC. However, important unanswered questions should be clarified: i) the optimal echocardiographic parameters; ii) the prognostic role of the novel TDI and STE methods; iii) utility of stress echocardiography (SE) to unmask myocardial dysfunction if not present at rest and its feasibility in LC patients; iv) the added value of the cardiac magnetic resonance imaging (CMR) and of cardiac biomarkers.

\section{Transthoracic echocardiography at rest}

Systolic function. It is now accepted that systolic dysfunction is mostly latent in LC patients [13-20]. But although left ventricular ejection fraction (LVEF) at rest is normal, there are subtle changes in myocardial function that might be detected by using TDI and STE [13-20]. LVEF by $2 \mathrm{DE}$ is the most widely used parameter for the left ventricular (LV) systolic function assessment. Most studies found that LVEF is normal in LC patients at rest [16-26]. According to the current echocardiographic guidelines, an LVEF of less than $52 \%$ in men and $54 \%$ in women, by $2 \mathrm{DE}$, suggests systolic dysfunction [27]. However, choosing a higher cut-off value (55-60\%) might be necessary for LC patients, due to their decreased afterload and increased preload, which could explain the normal LVEF values found in the majority of the studies $[16-26,28]$. Noteworthy, LVEF $\leq 60 \%$ was recently reported in a very large group of LC patients strongly associated with higher post-LT mortality rates in the MELD $\geq 20$ subgroup, suggesting that systolic dysfunction and severity of liver disease must be evaluated simultaneously in the pre-LT assessment protocol [28]. In conclusion, we recommend that the threshold for the diagnosis of LV systolic dysfunction in LC patients should be maintained at a higher cut-off $(\mathrm{LVEF}<55 \%)$, different from that of the 2019 consensus definition ( $\mathrm{LVEF}<50 \%)$.

Myocardial deformation evaluated by STE, already validated for the assessment of regional and global myocardial function [29], has been proposed for the assessment of early cardiac dysfunction in LC patients (Fig. 2). The advantage is that deformation is less load-dependent, when compared with standard 2DE. The American Society of Echocardiography (ASE) and the European Association of Cardiovascular Imaging (EACVI) guidelines define global longitudinal strain (GLS) less negative than $-16 \%$ as abnormal, GLS $-18 \%$ or greater as normal, and GLS $-16 \%$ to $-18 \%$ as borderline in adults $[6,27]$. Some STE studies have shown that LC patients had reduced GLS, despite still having normal LVEF $[18,19$, 30-32], while other studies found no differences in GLS in LC patients with different grades of LV diastolic, but not systolic dysfunction [17, 20]. In these circumstances, the novel 2019 CCM consensus includes GLS evaluation by STE in LC patients with preserved LVEF. Thus, diminished LVEF or diminished GLS in preserved LVEF, in the absence of known cardiac disease, should be used for the diagnostic of CCM (Fig. 1).

Regarding LT, few studies evaluated the changes in systolic function post-LT. Three of them showed a reduction in LVEF post-LT, but this decline was clinically insignificant $[2,23,33$, 34]. In one of these studies, although GLS remained within the normal range, there was a slight improvement of GLS at 18 months after LT [34]. In another study, comparison between the systolic response to stress before and after LT showed an improvement 9 months after transplantation, linking once more LC and CCM $[2,23]$.
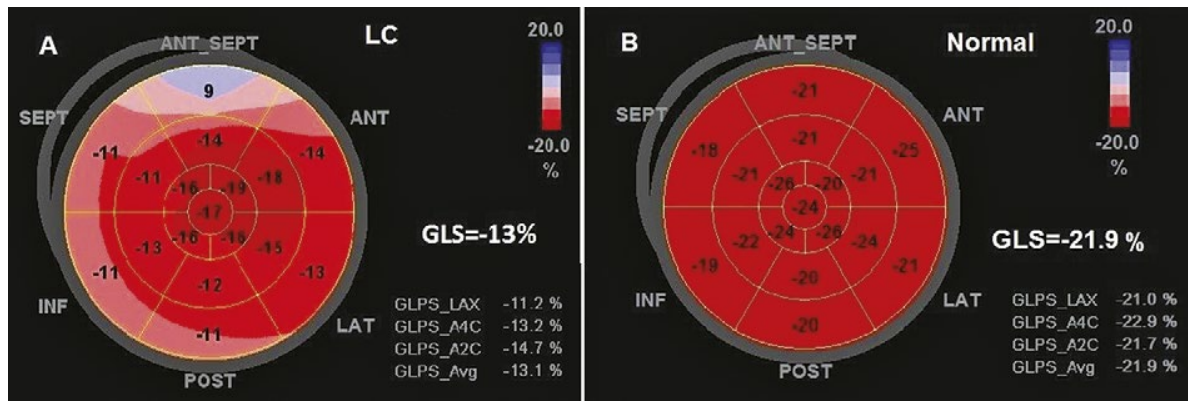

Fig. 2. Assessment of the left ventricular myocardial deformation by speckle-trackingechocardiography. A. Bull's eye reflecting global longitudinal deformation of the left ventricle (GLS) by speckle-tracking-echocardiography in a 55 year -old liver cirrhotic men, Child C, with preserved ejection fraction and diastolic dysfunction grade 2 . GLS $=-13 \%$ suggests a significantly decreased longitudinal deformation (red segments, normal deformation, light red decreased strain, white no deformation at all, and blue segments dyskinesia) B. Normal GLS in a normal subject with similar age and gender. 
In summary, GLS measured by STE might be a more accurate parameter for the detection of LV dysfunction as compared to LVEF. This parameter should be incorporated into the definition of CCM, as an alternative parameter in the context of preserved LVEF.

Although three-dimensional echocardiography (3DE) is now available in clinical practice, this imaging technique is highly dependent on image quality and the patient's capacity to hold their breath, which may limit its applicability in LC patients $[27,32]$. Contrast echocardiography can offer an improved endocardial border detection, and thus a more accurate evaluation of systolic function, especially in patients with poor acoustic windows. However, there are no data in LC patients.

Diastolic function. Diastolic dysfunction is in fact the cornerstone of CCM diagnosis. The prevalence of DD in LC patients is reported between 40 to $60 \%$ [16, 18, 19, 25]. All key mediators involved in the pathogenesis of CCM mainly affect the LV diastolic properties, with increased stiffness of the ventricular wall and decreased myocardial compliance and relaxation (Fig. 3). In the long-term, the consequences are subendothelial oedema, mild myocardial hypertrophy and fibrosis, followed by myocyte apoptosis [35-40]. Cirrhotic cardiomyopathy should thus be regarded as a particular type of $\mathrm{HF}$, difficult to be recognized because of the combination of low afterload and high preload conditions. However, similarly to the other types of HF, 2DE plays the key role in its evaluation. Echocardiographic changes in CCM include increased LV diameter, increased LV mass, thickened LV walls, increased indexed left atrial volume (LAVi) and DD, the latter considered to be a predictor of mortality not only in LC patients in general, but also in transplanted patients [41-43]. In the old definition of CCM, DD was expressed only as decreased peak E velocity (early rapid filling phase), prolonged deceleration time and isovolumetric relaxation time (IVRT), and an increased atrial contribution to the late ventricular filling (A wave) manifested as a decreased $\mathrm{E} / \mathrm{A}$ ratio. But since $\mathrm{E} / \mathrm{A}$ ratio is significantly dependent on loading conditions, for which LC patients have important variability, other parameters should be used for DD diagnosis. Moreover, the 2005 definition refers only to the impaired relaxation pattern of $\mathrm{DD}$, completely excluding all other types of DD [2,20] (Fig. 1).

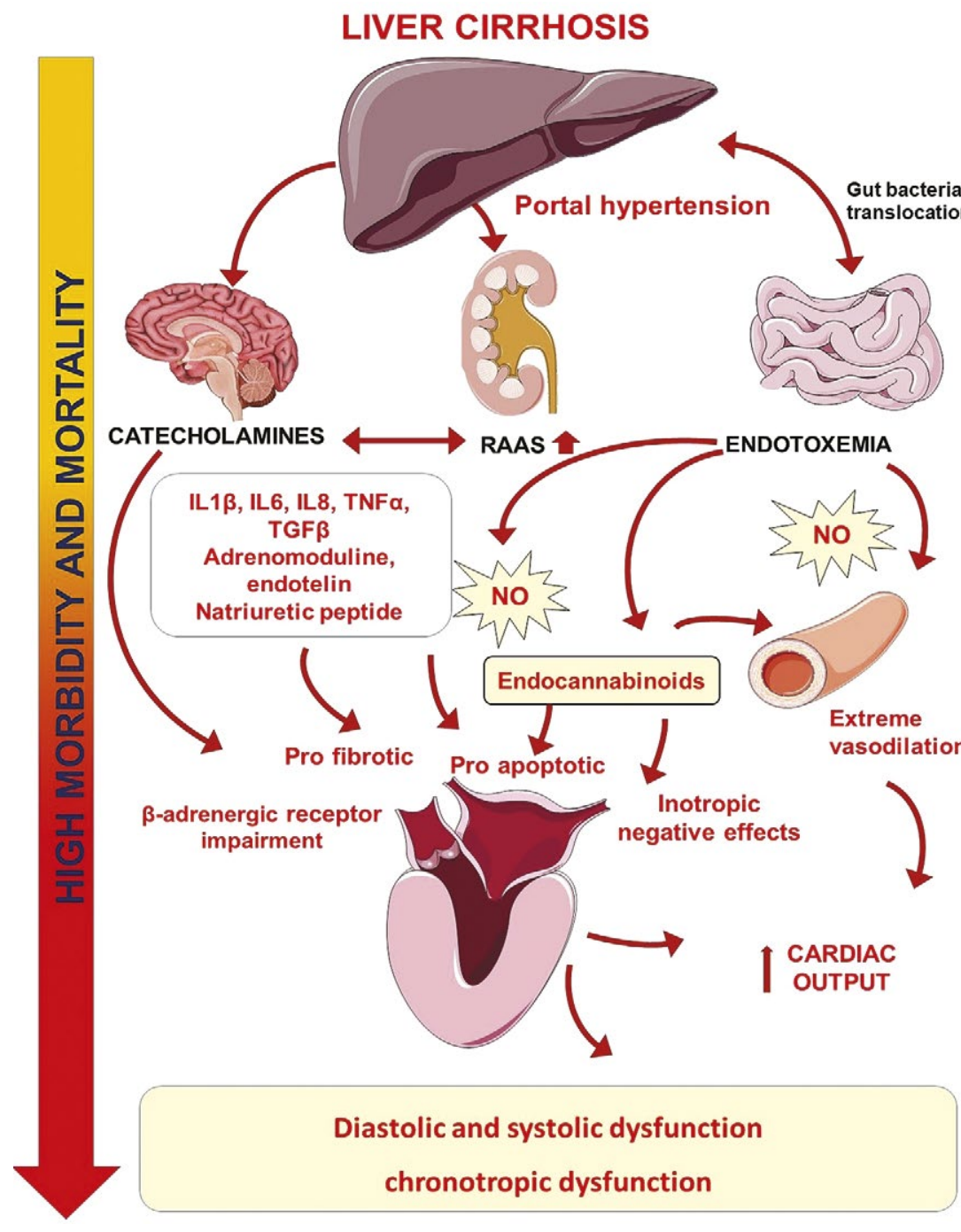

Fig. 3. Complex physiopathology of the cirrhotic cardiomyopathy. RAAS: renin angiotensin aldosterone system; IL: interleukin; TNF $\alpha$ : tumor necrosis factor $\alpha$; TGF $\beta$ : transforming growth factor $\beta$; NO: nitric oxide (modified from Rimbas et al. [2]). 
Tissue Doppler imaging is a well validated imaging technique for DD evaluation [21]. By TDI the diastolic tissue velocity of mitral annulus ( $\left.E^{\prime}\right)$ can be measured. At present, the E/E' ratio is used to estimate LVFP, as recommended by the ASE guidelines for the evaluation of DD [21]. Different studies suggested that the presence of DD in LC patients, assessed by using ASE guidelines, is related to mortality, and E/E' ratio is an independent predictor of mortality [41, 42, 44]. In the largest study to date, Cesari et al. [45] also proved that the E/E ratio can be used accurately to estimate LVFP in LC patients. These data convinced the experts to include this parameter in the novel consensus definition of CCM. However, a recent meta-analysis reported insufficient evidence to support $\mathrm{E} / \mathrm{E}$ ratio as a reliable parameter for the quantification of LVFP in patients with HF with preserved ejection fraction (HFpEF), concluding that this parameter should not be used alone for the estimation of LVFP [46]. We previously demonstrated that another parameter, the IVRT/TE-E' ratio $<2.45$ (time from onset of $\mathrm{E}$ to $\mathrm{E}^{\prime}$ wave), instead of $\mathrm{E} / \mathrm{E}^{\prime}$ ratio, should be used for a better estimation of LVFP [20]. It was found to be independent of loading conditions, and might predict mortality better than E/E' ratio in the DD subgroup [20].

An increase in LAVi was interpreted in some studies as a marker of DD in LC patients [37]. Other studies suggested that the increased LAVi is rather related to the high preload and should not be used as a single marker of DD [2, 30, 32]. However, LAVi is a mandatory measurement for the assessment of DD in the 2016 ASE guideline [21]. Besides, left atrium (LA) reservoir function evaluated by STE seems to correlate better with LVFP than LAVi or E/E' ratio in LC patients, and might be used to improve DD quantification in these patients [30,32] (Fig. 4). This is also specified in the 2019 consensus definition, to be used in unclassifiable cases, but without a clear cut off.

Another important issue is the severity of DD in LC patients, usually classified from mild (grade 1) to severe (grade 3). Many recent studies have shown that the majority of the cirrhotic patients have mild or moderate DD, while only a minority of them have a restrictive pattern $[20,45$, 47]. Importantly, DD severity correlates with the severity of the liver disease $[42,47]$. However, this grading system has been incorporated only in the updated definition of CCM [6]. The ASE algorithm for DD quantification in general cardiac pathology, proposed by the experts, includes TDI mitral annulus velocities, PW Doppler mitral inflow, E/E' ratio, tricuspid velocity (TV) and LAVi [21]. It recommends four variables for identifying $\mathrm{DD}$, and specifies their cut-off values: annular septal $E^{\prime}<7 \mathrm{~cm} / \mathrm{sec}$ or lateral $E^{\prime}<10 \mathrm{~cm} / \mathrm{sec}$, average E/E'ratio $>14, \mathrm{LAVi}>34 \mathrm{~mL} / \mathrm{m}^{2}$ and elevated pulmonary artery pressure predicted by $\mathrm{TV}>2.8 \mathrm{~m} / \mathrm{sec}$ [21]. If three variables or more are abnormal, DD is present, and $\mathrm{E}$-wave and $\mathrm{E} / \mathrm{A}$ ratio determines its severity or grading. If three or more variables are normal, DD is absent. When only two of the variables are abnormal, DD cannot be declared [21]. In these uncertain cases, it was recently suggested that LA reservoir strain (LASr), evaluated by STE, might improve DD detection, when all other parameters give conflicting information. Because the primary function of the LA is to modulate LV filling, it seems reasonable that functional LA changes will become evident at the earliest stages of LVDD, even before volumetric changes
[48-50]. Measurement of LASr seems to improve the diagnostic accuracy of both DD and HFpEF algorithms [48]. A cut off value of LASr $<35 \%$ suggests functional abnormality of LA even in normal LAVi [48]. Moreover, cut-off values corresponding to each DD grade were recently provided by a panel of experts, LASr between 35 to $24 \%$ for grade 1, between 24 to $19 \%$ for grade 2 , and LASr $<19 \%$ for grade 3 [48]. LASr $<20 \%$ was invasively validated as an optimal parameter to detect elevated LVFP. The improvement of DD classification using LASr compared to the guidelines was found more pronounced in subjects with normal LV function [51]. In summary, in LC patients, in which LAVi is almost always higher than normal, due to high preload conditions, the use of LASr to estimate elevated LVFP might be more accurate than the current 2016 DD guidelines approach. Thus, the introduction of LASr into the non-invasive assessment of LV diastolic function might improve the detection of elevated LVFP in LC.

The novel 2019 CCM consensus proposed a new adapted algorithm for detection and grading of DD, unpublished yet, and without validation [6]. To illustrate difficulties in DD quantification in LC patients, Cesari et al. [45] used all the classification systems for DD evaluation proposed so far by the ASE/EACVI in 2009 and 2016, and by the 2016 Thorax Centre algorithm $[21,52,53]$. It is the largest prospective study to date (115 LC patients followed up for at least 6 years for fatal outcome), aimed to establish the prognostic value of different echocardiographic parameters in addition to clinical and main hemodynamic parameters. Noteworthy, the difference in the prevalence of LVDD was significant between algorithms, emphasizing the idea of a major methodological gap in the quantification of DD in LC patients [54]. Thus, it is obvious why it is so important to reach a better definition for the quantification of the rest myocardial dysfunction, as a reference standard, and to define its contribution to mortality in LC patients, transplanted or not. It is essential to identify echocardiographic predictors of a worse outcome, especially since elevated LVFP cannot be assessed by cardiac catheterization in the majority of LC patients.

The 2016 ASE/EACVI algorithm was found to be more user-friendly and efficient than the 2009 algorithm, and has demonstrated that it can provide accurate estimates of LVFP in the majority of patients when compared with invasive measurements $[50,51]$. Therefore, we suggest that the 2016 ASE/EACVI algorithm for the detection and grading of DD, already invasively validated, should be incorporated into the evaluation protocol for CCM, instead of the recently proposed DD grading from the CCM consensus, which has not yet been validated in other cardiovascular diseases [6].

\section{Stress echocardiography}

Some LC patients have symptoms such as dyspnoea only during exercise. Accordingly, $2 \mathrm{DE}$ at rest could be insufficiently sensitive to identify cardiac abnormalities in these patients [2]. Stress echocardiography is used for the detection of chronotropic dysfunction, as part of the cardiovascular risk assessment of LC patients before LT. This phenomenon is diagnosed when the achieved heart rate (HR) is less than $85 \%$ of maximal predicted $\mathrm{HR}$, and represents a strong independent predictor of major cardiovascular events. It occurs in $26-37 \%$ 
of the end-stage LC patients undergoing SE [55], and can be explained by down-regulation and desensitization of the betaadrenergic receptors in the sino-atrial node [56]. However, its predictive value for the presence of CCM is not well established [57-59].

The current definition specifies that although the LVEF is normal at rest, contractile response to stress is impaired. Stress echocardiography might be used in patients with LC because it allows a dynamic assessment of the myocardial function under physiological or pharmacological stress, in order to unmask cardiac dysfunction, similarly to clinical stressful conditions such as major surgical interventions [5862]. Stress echocardiography can be done, either by exercise - the modality of choice for patients capable of physical effort, or by pharmacological stress - with dobutamine [57, 64]. However, the pharmacological stress might not reproduce the complex haemodynamic and neurohormonal changes induced by exercise. By using a supine bicycle, the echocardiographic acquisition can be performed throughout the test. The abnormal LV response during exercise consists of a failure to augment ejection fraction by $>5 \%$ in response to stress [32, 63]. In patients with normal LVFP at rest, the SE may uncover an increased LVFP in response to exercise, due to impaired diastolic reserve, identified by an increase in E/E' ratio [60]. A conclusive negative SE have a high negative predictive value for cardiac events after LT [55].

Measurement of the E/E' ratio during exercise is feasible and has been invasively validated for the estimation of raised LVFP, an E/E'>15 accurately identifying increased LVFP (>15 $\mathrm{mmHg}$ [ 64,65$]$. However, there are conflicting data regarding the utility of SE in CCM diagnosis, mostly generated by the inability to achieve the predicted $\mathrm{HR}$ target $[11,57,58]$. Up to $56 \%$ of SE studies in LC patients have been reported as inconclusive [9]. Barbosa et al. [57] suggested SE as an important tool for the diagnosis of CCM. Their findings might explain the development of acute pulmonary oedema after trans-jugular intrahepatic portosystemic shunt insertion and LT, as both interventions generate a sudden increase in preload and, consequently, a rise in LVFP.

Stress echocardiography is now considered very useful in the HFpEF diagnosis, as a functional marker, in the most recent published guidelines [65]. Stress echocardiography should be considered abnormal if average E/E' ratio at peak stress increases to $\geq 15$, with or without a peak $\mathrm{TV}>3.4 \mathrm{~m} / \mathrm{s}$ [65]. However, the recent CCM definition consensus does not include SE in the diagnosis algorithm, and reserves it only for research purposes [6]. We consider that SE should be part of the diagnosis protocol in all uncertain cases, as the CCM is a particular type of HF with low afterload and high preload.

In summary, taking into account all these data about the diagnostic potential and limitations of the old and novel echocardiographic parameters, we suggest that the cardiac evaluation in LC should be improved. Since each parameter alone has some potential limitations, the diagnosis of DD should not rely on a single measurement and rather a multiparameter approach should be used, in a step-by-step fashion, in order to better classify the severity of DD and, importantly, to estimate LVFP in the context of high preload conditions. Also, SE should be used in selected cases to unmask DD. Therefore, we propose a new step-by-step algorithm for the evaluation of LC patients (Figs. 5 and 6), which takes into account all new published data about CCM, and all new guidelines from the echocardiographic evaluation in the field of HF, discussed above.

\section{Cardiac magnetic resonance}

Cardiac magnetic resonance imaging is considered as a "gold standard" for accurate assessment of the LVEF, chamber volumes, myocardial fibrosis and oedema, prior to the onset of LV dysfunction. The presence of late gadolinium enhancement was found in LC patients, regardless of the cause of liver disease, even if it appears more pronounced in patients with alcoholic LC $[2,6,66]$. Studies using CMR in cirrhotic patients have shown increased LAVi, LV end diastolic volume, and LV hypertrophy [66]. Structural changes in CCM were found to be similar to the findings in patients with myocarditis, with a non-specific patchy distribution [67]. However, the applicability of CMR for CCM diagnosis is low, mainly due to a non-specific pattern.

\section{Electrophysiological changes}

At present, electrocardiographic (ECG) findings are of limited value in CCM [6]. The pathophysiologic consequences of LC are prolongation of the QT interval, chronotropic
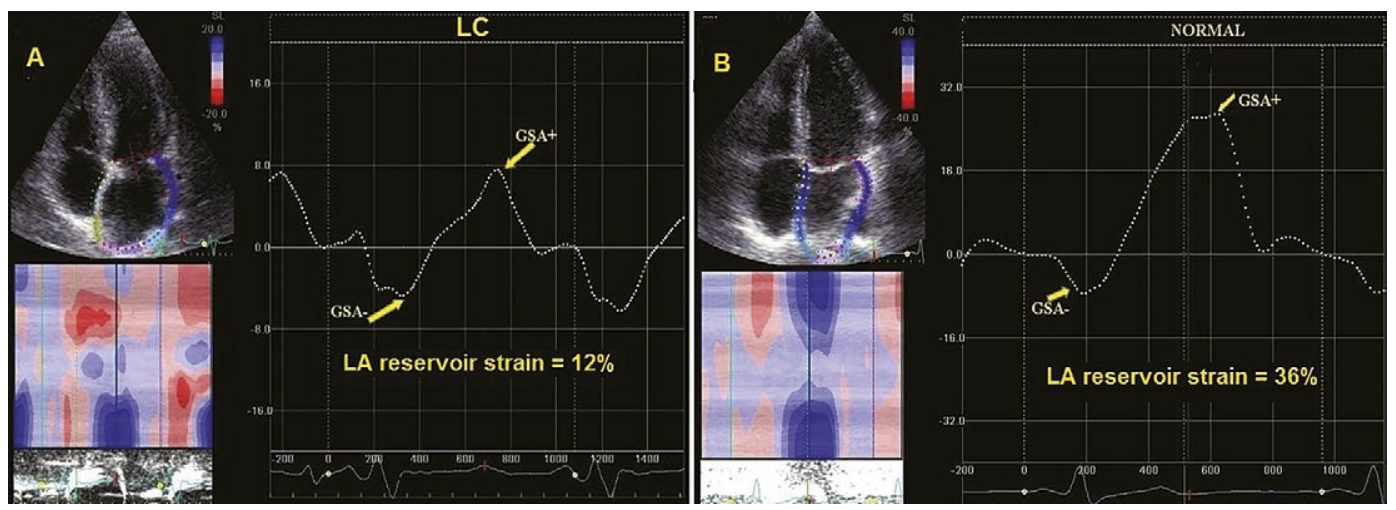

Fig. 4. Evaluation of the left atrial (LA) myocardial deformation by speckle-tracking-echocardiography A. Decreased LA booster (GSA-), conduit (GSA+), and reservoir strain (the sum of GSA- and GSA+ in absolute values), in a female patient with liver cirrhosis, 52 years old. LA reservoir, booster, and conduit strains are assessed from apical 4-chamber view. B. Normal atrial deformation in a normal subject with similar age and gender. 


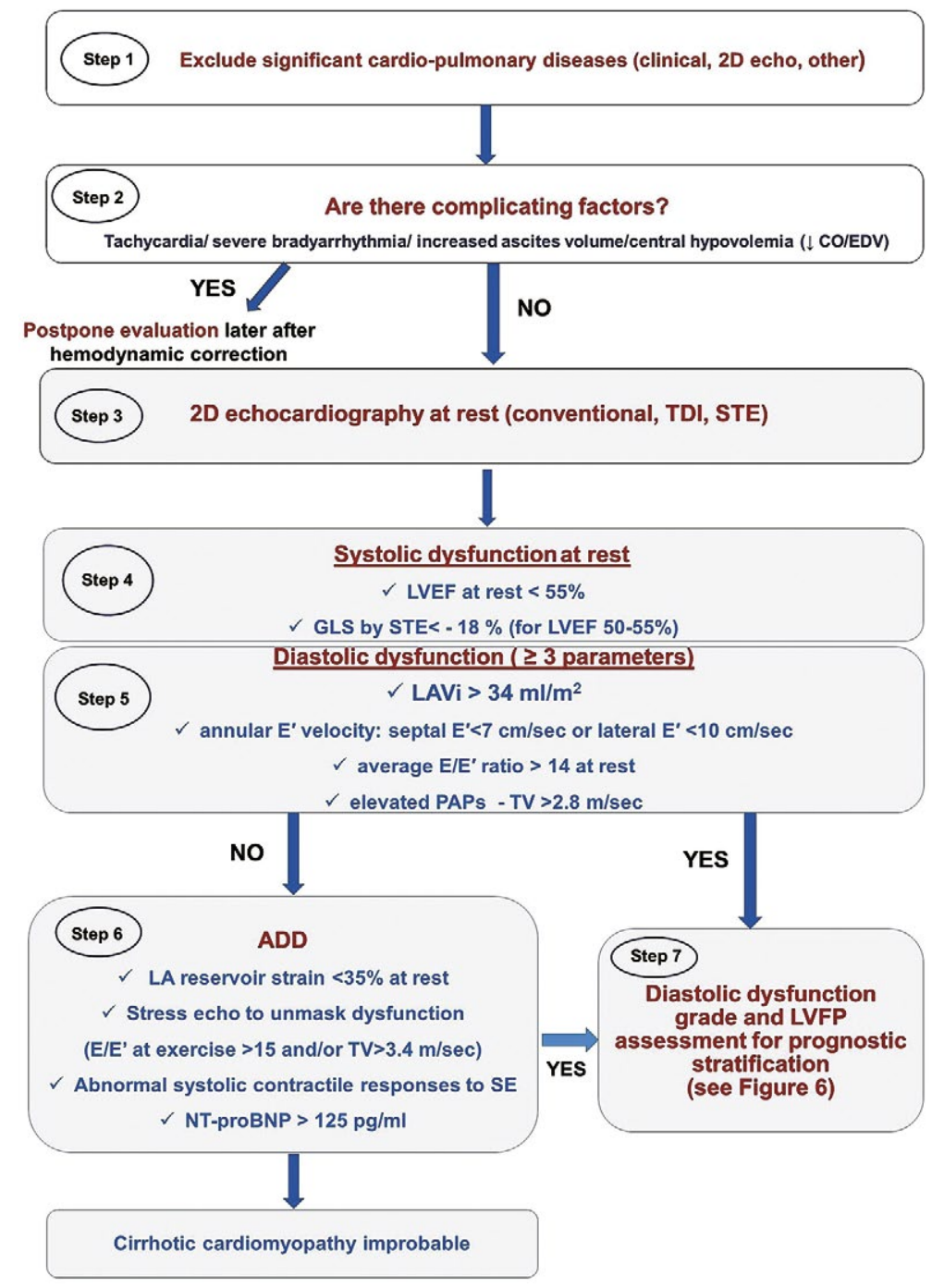

Fig. 5. Proposed stepwise evaluation for diagnosis of cirrhotic cardiomyopathy 2D: two-dimensional echocardiography; CO: cardiac output; EDV: end-diastolic volume; TDI: tissue Doppler imaging; STE: speckle-tracking-echocardiography; LVEF: left ventricular ejection fraction; GLS: global longitudinal strain by STE; LAVi: indexed left atrial volume; E': early diastolic velocity from the pulsed-wave tissue Doppler imaging; E: peak velocity flow in early diastole; TV: tricuspid velocity; sPAP, systolic pulmonary artery pressure; SE: stress echocardiography; NTproBNP: N-terminal prohormone of brain natriuretic peptide; LVFP: left ventricular filling pressure.

dysfunction, and electromechanical uncoupling. Prolongation of the QT interval (>440 $\mathrm{msec}$ ) is the most common ECG finding, with a prevalence of 37-84\% [68]. Other ECG abnormalities in LC patients are atrial and ventricular premature contractions, bundle branch blocks, and ST segment depression in more advanced stages. 24-hour Holter monitoring has better sensitivity to detecting arrhythmia, and can reveal subclinical anomalies $[69,70]$. In some studies, the prolonged QTc interval of more than $440 \mathrm{msec}$ correlated with 1-year mortality, but only in patients with DD $[20,71]$. Nonetheless, all studies showed significant improvement of QTc after LT, including normalization in more than $80 \%$ of the patients [33], but its utility in the prediction of poor outcomes remains controversial [6]. Therefore, the novel consensus did not consider anymore ECG abnormalities in the CCM diagnostic criteria.

\section{Cardiac biomarkers}

Recent studies showed that cardiac biomarkers, especially troponin I (TpI), BNP, and NT-proBNP are elevated in LC patients [41]. Troponin I level was recently found to significantly correlate with the severity of LC and overall mortality $[41,70]$. However, a cut-off value for the diagnosis of CCM and for the prognosis assessment in LC patients was not yet established.

Since CCM is in fact a particular type of HF, BNP and its prohormone NT-proBNP might play a major role in its diagnosis. Both BNP and NT-proBNP are natriuretic peptides, primarily secreted by the cardiac ventricles in response to increased LVFP [72]. Therefore, patients with LC usually have elevated levels of BNP/NT-proBNP, which could result from stretching of the cardiomyocytes from volume overload, 


\section{LEFT VENTRICULAR FILLING PRESSURE ASSESSMENT IN}

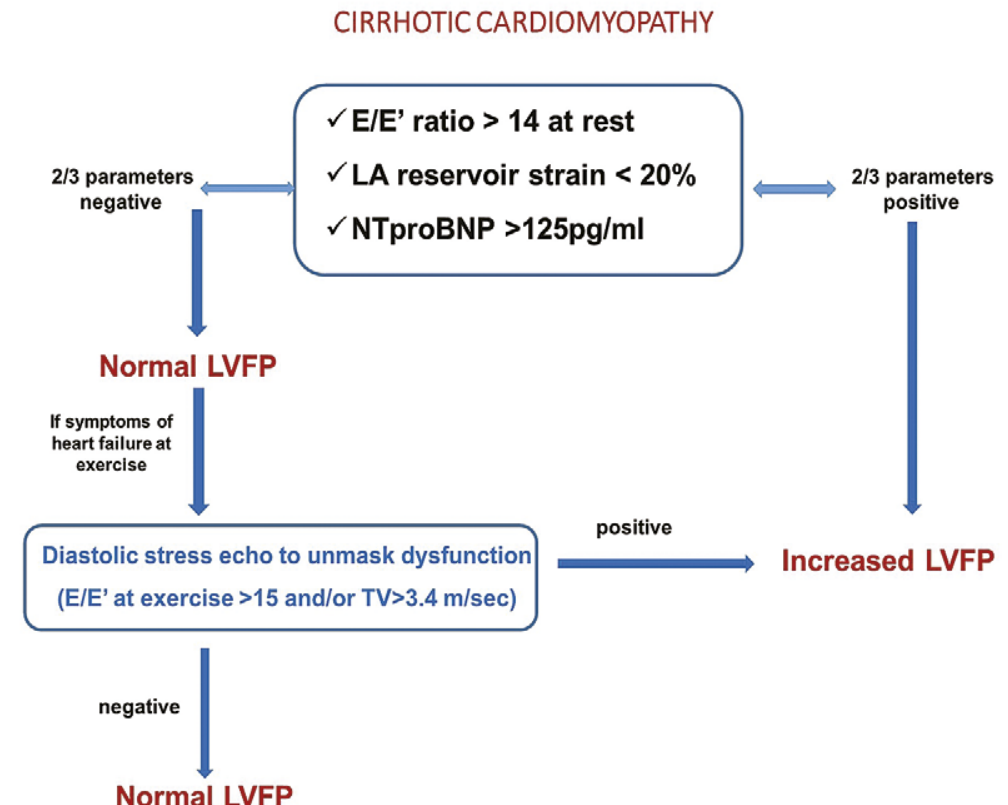

Fig. 6. Proposed algorithm for left ventricular filling pressure assessment in liver cirrhosis patients. E/E' ratio: ratio between E, peak velocity flow in early diastole, and E', early diastolic velocity from the pulsed-wave tissue Doppler imaging; LA: left atrial; NTproBNP: N-terminal prohormone of brain natriuretic peptide; LVFP: left ventricular filing pressure; TV: tricuspid velocity.

but also from insufficient clearance, noticed especially in decompensated cirrhosis [72-74]. NT-proBNP was found to be a better indicator for early cardiac dysfunction than BNP because of its stability and longer biological half-life [73]. Both of them significantly correlate with interventricular septal thickness, LAVi, E/E' ratio, and the presence of DD [74-76]. Most importantly, their increased levels before transplantation appear to normalize afterwards [77]. Their cut-off values for chronic HF diagnosis are generally considered $35 \mathrm{pg} / \mathrm{ml}$ for $\mathrm{BNP}$, and $125 \mathrm{pg} / \mathrm{ml}$ for NT-pro-BNP in patients without LC [78]. However, since the levels of these biomarkers seem to be higher in cirrhosis, there is a need for a conclusive cut-off value to rule-out patients who are unlikely to have CCM. One study found that LC patients with plasma levels of NT-proBNP of more than $290 \mathrm{pg} / \mathrm{ml}$ are at an increased risk of CCM and should be referred for specific cardiac evaluation, but this study used the old definition criteria [79]. Moreover, BNP levels before LT represent an independent predictor of early mortality after transplantation, with an excellent negative predictive value [79]. A study on $525 \mathrm{LT}$ recipients showed that the level of BNP before LT was higher in the non-survival group $(114 \mathrm{pg} / \mathrm{ml})$ versus the survival group after LT $(56 \mathrm{pg} / \mathrm{ml})(\mathrm{p}<0.001)$. A value higher than $136 \mathrm{pg} / \mathrm{ml}$ was associated with an increased mortality after LT with a specificity of $83.5 \%$ [80].

Galectin-3, copeptin, soluble suppression of tumorgenicity-2 (ST-2, member of the interleukin family) are newly investigated biomarkers for myocardial injury, inflammatory and fibrotic cardiac remodelling $[6,81]$. But galectin-3 and soluble ST-2 have also been shown to be markers of liver inflammation and fibrosis, which may limit their applicability as diagnostic criteria for CCM. Besides, the prognostic role and the cut- off values for all these biomarkers in LC patients are not established.

\section{CONCLUSIONS}

Cirrhotic cardiomyopathy should be regarded as a particular HF type, characterized mainly by diastolic, but also systolic dysfunction. Cirrhotic cardiomyopathy contributes to the high cardiovascular morbidity and mortality related to LT and to the overall prognosis of the patient. Although a novel consensus definition was recently published, there are still unanswered questions and points to be improved, in order to efficiently diagnose this condition. With the remarkable developments in cardiac imaging, an improvement of the current definition criteria is urgently required, including TDI and STE parameters, and also stress echocardiography in specific situations, in order to unmask silent myocardial dysfunction at rest. Diastolic dysfunction and LVFP assessment should be based on a multi-parameter approach, in order to properly identify patients at risk for worse outcomes especially after LT. We proposed a new step-by-step algorithm for the CCM diagnosis, which takes into account all recent published data and new guidelines from general cardiology. Future studies, using a new updated algorithm, are required to establish which parameters serve better to diagnose and monitor the cardiac dysfunction in cirrhotic patients, which parameters predict worst prognosis after LT, and if identifying cardiac dysfunction in early stages has an important prognostic value.

Conflicts of interest: None to declare. 
Authors' contribution: R.C.R.: conceived and drafted the study, wrote the manuscript, revised it critically for important intellectual content. M.R. and C.P. substantially contributed to the conception and drafting the study and revising it critically for important intellectual content. A.M.C. and L.M.L collected and analysed the data. D.V.: designed the study and critically revised it for important intellectual content. All the authors approved the final version of the manuscript.

\section{REFERENCES}

1. Hoyert DL, Xu J. Deaths: preliminary data for 2011. Natl Vital Stat Rep 2012;61:1-51

2. Rimbas RC, Chitroceanu AM, Vinereanu D. Cirrhotic Cardiomyopathy. In: Radu-Ionita F Pyrsopoulos NT, Jinga M, Tintoiu IC, Sun Z, Bontas E. (eds). Liver diseases. A Multidisciplinary Textbook. Springer Nature, Switzerland, 2020:305-318.

3. Madhwal S, Atreja A, Albeldawi M, Lopez R, Post A, Costa MA. Is liver transplantation a risk factor for cardiovascular disease? A meta-analysis of observational studies. Liver Transpl 2012;18:1140-1146. doi:10.1002/ lt.23508

4. European Association for the Study of the Liver. EASL Clinical Practice Guidelines: Liver transplantation European Association for the Study of the Liver. J Hepatol 2016;64:433-485. doi:10.1016/j.jhep.2015.10.006

5. Martin P, DiMartini A, Feng S, Brown R Jr, Fallon M. Evaluation for Liver Transplantation in Adults: 2013 Practice Guideline by the American Association for the Study of Liver Diseases and the American Society of Transplantation. Hepatology 2014;59:1144-1165. doi:10.1002/hep.26972

6. Izzy M, VanWagner LB, Lin G, et al. Redefining cirrhotic cardiomyopathy for the modern era. Hepatology 2020;71:334-345 doi:10.1002/ hep. 30875

7. Mocarzel LOC, Rossi MM, Miliosse BM, Lanzieri PG, Gismondi RA. Cirrhotic cardiomyopathy: a new clinical phenotype. Arq Bras Cardiol 2017;108:564-568. doi:10.5935/abc.20170066

8. Kazankov K, Holland-Fischer P, Andersen NH, et al. Resting myocardial dysfunction in cirrhosis quantified by tissue Doppler imaging. Liver Int 2011;31:534-540. doi:10.1111/j.1478-3231.2011.02468.x

9. Ripoll C, Yotti R, Bermejo J, Benares R. The heart in liver transplantation. J Hepatol 2011;54:810-822. doi:10.1016/j.jhep.2010.11.003

10. Zardi EM, Abbate A, Zardi DM, et al. Cirrhotic cardiomyopathy. J Am Coll Cardiol 2010;56:539-549. doi:10.1016/j.jacc.2009.12.075

11. Zaky A, Bendjelid K. Appraising cardiac dysfunction in liver transplantation: An ongoing challenge. Liver Int 2015;35:12-29. doi:10.1111/liv.12582

12. Møller S, Henriksen JH. Cirrhotic cardiomyopathy. J Hepatol 2010;53:179-190. doi:10.1016/j.jhep.2010.02.023

13. Møller S, Hove JD, Dixen U, Bendtsen F. New insights into cirrhotic cardiomyopathy. Int J Cardiol 2013;167:1101-1108. doi:10.1016/j. ijcard.2012.09.089

14. Dowsley TF, Bayne DB, Langnas AN, et al. Diastolic dysfunction in patients with end-stage liver disease is associated with development of heart failure early after liver transplantation. Transplantation 2012;94:646-651. doi:10.1097/TP.0b013e31825fof97

15. Alexopoulou A, Papatheodoridis G, Pouriki S, et al. Diastolic myocardial dysfunction does not affect survival in patients with cirrhosis. Transpl Int 2012;25:1174-1181. doi:10.1111/j.1432-2277.2012.01547.x

16. Merli M, Calicchia A, Ruffa A, et al. Cardiac dysfunction in cirrhosis is not associated with the severity of liver disease. Eur J Intern Med 2013;24:172-176. doi:10.1016/j.ejim.2012.08.007
17. Nazar A, Guevara M, Sitges $M$, et al. Left ventricular function assessed by echocardiography in cirrhosis: relationship to systemic hemodynamic and renal dysfunction. J Hepatol 2013;58:51-57. doi:10.1016/j. jhep.2012.08.027

18. Sampaio F, Pimenta J, Bettencourt N, et al. Systolic and diastolic dysfunction in cirrhosis: A tissue-Doppler and speckle tracking echocardiography study. Liver Int 2013;33:1158-1165. doi:10.1111/ liv. 12187

19. Sampaio F, Pimenta J, Bettencourt N, et al. Systolic dysfunction and diastolic dysfunction do not influence medium-term prognosis in patients with cirrhosis. Eur J Intern Med 2014;25:241-246. doi:10.1016/j. ejim.2014.01.011

20. Rimbaş RC, Baldea SM, Guerra RDGA, et al. New definition criteria of myocardial dysfunction in patients with liver cirrhosis: A speckle tracking and tissue doppler imaging study. Ultrasound Med Biol 2018;44:562-574. doi:10.1016/j.ultrasmedbio.2017.11.013

21. Nagueh SF, Smiseth OA, Appleton CP, et al. Recommendations for the evaluation of left ventricular diastolic function by echocardiography: An update from the American Society of Echocardiography and the European Association of Cardiovascular Imaging. J Am Soc Echocardiogr 2016;29:277-314. doi:10.1016/j.echo.2016.01.011

22. Horvatits T, Drolz A, Rutter K, Roedl K, Kluge S, Fuhrmann V. Hepatocardiac disorders: Interactions between two organ systems. Med Klin Intensivmed Notfmed 2016;111:447-452. doi:10.1007/s00063-0150043-x

23. Liu H, Jayakumar S, Traboulsi M, Lee SS. Cirrhotic cardiomyopathy: Implications for liver transplantation. Liver Transpl 2017;23:826-835. doi:10.1002/lt.24768

24. Torregrosa M, Aguadé S, Dos L, et al. Cardiac alterations in cirrhosis: reversibility after liver transplantation. J Hepatol 2005;42:68-74 doi:10.1016/j.jhep.2004.09.008

25. Gassanov N, Caglayan E, Semmo N, Massenkeil G, Er F. Cirrhotic cardiomyopathy: A cardiologist's perspective. World J Gastroenterol 2014;20:15492-15498. doi:10.3748/wjg.v20.i42.15492

26. Mota VG, Markman Filho B. Echocardiography in chronic liver disease: systematic review. Arq Bras Cardiol 2013;100:376-385. doi:10.5935/ abc. 20130047

27. Lang RM, Badano LP, Mor-Avi V, et al. Recommendations for cardiac chamber quantification by echocardiography in adults: An update from the American Society of Echocardiography and the European Association of Cardiovascular Imaging. J Am Soc Echocardiogr 2015;28:1-39.e14. doi:10.1016/j.echo.2014.10.003

28. Kwon HM, Moon YJ, Jung KW, et al. Appraisal of cardiac ejection fraction with liver disease severity: Implication in post-liver transplantation mortality. Hepatology 2020;71:1364-1380. doi:10.1002/ hep.30913

29. Mor-Avi V, Lang RM, Badano LP, et al. Current and evolving echocardiographic techniques for the quantitative evaluation of cardiac mechanics: ASE/EAE consensus statement on methodology and indications endorsed by the Japanese Society of Echocardiography. Eur J Echocardiogr 2011;12:167-205. doi:10.1016/j.echo.2011.01.015

30. Altekin RE, Caglar B, Kaarakas MS, Ozel D, Deger N, Demir I Evaluation of subclinical left ventricular systolic dysfunction using two-dimensional speckle-tracking echocardiography in patients with non-alcoholic cirrhosis. Hellenic J Cardiol 2014;55:402-410.

31. Krag A, Bendtsen F, Mortensen C, Henriksen JH, Møller S. Effects of a single terlipressin administration on cardiac function and perfusion in cirrhosis. Eur J Gastroenterol Hepatol 2010;22:1085-1092. doi:10.1097/ MEG.0b013e32833a4822 
32. Sampaio F, Pimenta J. Left ventricular function assessment in cirrhosis: Current methods and future directions. World J Gastroenterol 2016;22:112-125. doi:10.3748/wjg.v22.i1.112

33. Izzy M, Oh J, Watt KD. Cirrhotic cardiomyopathy after transplantation: neither the transient nor innocent bystander. Hepatology 2018;68:20082015. doi:10.1002/hep.30040

34. Chen Y, Chan AC, Chan SC, et al. A detailed evaluation of cardiac function in cirrhotic patients and its alteration with or without liver transplantation. J Cardiol 2016;67:140-146. doi:10.1016/j. jjcc.2015.08.001

35. Liu H, Gaskari SA, Lee SS. Cardiac and vascular changes in cirrhosis: pathogenic mechanisms. World J Gastroenterol 2006;12:837-842.

36. Wong F. Cirrhotic cardiomyopathy. Hepatol Int 2009;3:294-304. doi:10.1007/s12072-008-9109-7

37. Møller S, Bernardi M. Interactions of the heart and the liver. Eur Heart J 2013;34:2804-2811. doi:10.1093/eurheartj/eht246

38. Bolognesi M, Sacerdoti D, Piva A, et al. Carbon monoxide-mediated activation of large conductance calcium-activated potassium channels contributes to mesenteric vasodilatation in cirrhotic rats. J Pharmacol Exp Ther 2007;321:187-194. doi:10.1124/jpet.106.116665

39. Baik SK, Fouad TR, Lee SS. Cirrhotic cardiomyopathy. Orphanet J Rare Dis 2007;2:15. doi:10.1186/1750-1172-2-15

40. Wiese S, Hove JD, Bendtsen F, Møller S. Cirrhotic cardiomyopathy: pathogenesis and clinical relevance. Nat Rev Gastroenterol Hepatol 2014;11:177-186. doi:10.1038/nrgastro.2013.210

41. Ruiz-del-Arbol L, Monescillo A, Arocena C, et al. Circulatory function and hepatorenal syndrome in cirrhosis. Hepatology 2005;42:439-447. doi:10.1002/hep.20766

42. Ruiz-Del-Arbol L, Achecar L, Serradilla R, et al. Diastolic dysfunction is a predictor of poor outcomes in patients with cirrhosis, portal hypertension and a normal creatinine. Hepatology 2013;58:1732-1741. doi:10.1002/hep.26509

43. Carvalheiro F, Rodrigues C, Adrego T, et al. Diastolic dysfunction in liver cirrhosis: Prognostic predictor in liver transplantation? Transplant Proc 2016;48:128-131. doi:10.1016/j.transproceed.2016.01.010

44. Cazzaniga M, Salerno F, Pagnozzi G, et al. Diastolic dysfunction is associated with poor survival in patients with cirrhosis with transjugular intrahepatic portosystemic shunt. Gut 2007;56:869-875. doi:10.1136/ gut.2006.102467

45. Cesari M, Frigo AC, Tonon M, Angeli P. Cardiovascular predictors of death in patients with cirrhosis. Hepatology 2018;68:215-223. doi: $10.1002 /$ hep. 29520

46. Sharifov OF, Schiros CG, Aban I, Denney TS, Gupta H. Diagnostic accuracy of tissue Doppler index E/e for evaluating left ventricular filling pressure and diastolic dysfunction/heart failure with preserved ejection fraction: A systematic review and metanalysis. J Am Heart Assoc 2016;5:e002530. doi:10.1161/JAHA.115.002530

47. Naqvi IH, Mahmood K, Naeem M, Vashwani AS, Ziaullah S. The heart matters when the liver shatters! Cirrhotic cardiomyopathy: Frequency, comparison, and correlation with severity of disease. Prz Gastroenterol 2016;11:247-256. doi:10.5114/pg.2016.57962

48. Thomas L, Marwick TH, Popescu BA, Donal E, Badano LP. Left atrial structure and function, and left ventricular diastolic dysfunction. J Am Coll Cardiol 2019;73:1961-1977. doi:10.1016/j.jacc.2019.01.059

49. Badano LP, Kolias TJ, Muraru D, et al Standardization of left atrial, right ventricular, and right atrial deformation imaging using two-dimensional speckle tracking echocardiography: a consensus document of the EACVI/ ASE/Industry Task Force to standardize deformation imaging. Eur Heart J Cardiovasc Imaging 2018;19:591-600. doi:10.1093/ehjci/jey042
50. Balaney B, Medvedofsky D, Mediratta A, et al. Invasive validation of the echocardiographic assessment of left ventricular filling pressures using the 2016 diastolic guidelines: Head-to-head comparison with the 2009 guidelines. J Am Soc Echocardiogr 2018;31:79-88. doi:10.1016/j. echo.2017.09.002

51. Singh A, Medvedofsky D, Mediratta A, et al. Peak left atrial strain as a single measure for the non-invasive assessment of left ventricular filling pressures. Int J Cardiovasc Imaging 2019;35:23-32. doi:10.1007/ s10554-018-1425-y

52. Nagueh SF, Appleton CP, Gillebert TC, et al. Recommendations for the evaluation of left ventricular diastolic function by echocardiography. Eur J Echocardiogr 2009;10:165-193. doi:10.1093/ejechocard/jep007

53. van Dalen BM, Strachinaru M, van der Swaluw J, Geleijnse ML. A simple, fast and reproducible echocardiographic approach to grade left ventricular diastolic function. Int J Cardiovasc Imaging 2016;32:743752. doi:10.1007/s10554-015-0832-6

54. Rimbaş RC, Rimbaş M, Vinereanu D. Letter to the Editor: Echocardiographic predictors of a worse outcome in patients with liver cirrhosis: What should we assess? Hepatology 2019;69:2714-2715. doi:10.1002/hep.30584

55. Umphrey LG, Hurst RT, Eleid MF, et al. Preoperative dobutamine stress echocardiographic findings and subsequent short-term adverse cardiac events after orthotopic liver transplantation. Liver Transpl 2008;14:886892. doi:10.1002/lt.21495

56. Dahl EK, Møller S, Kjær A, Petersen CL, Bendtsen F, Krag A. Diastolic and autonomic dysfunction in early cirrhosis: a dobutamine stress study. Scand J Gastroenterol 2014;49:362-372. doi:10.3109/00365521.2013.86 7359

57. Barbosa M, Guardado J, Marinho C, et al. Cirrhotic cardiomyopathy: Isn't stress evaluation always required for the diagnosis? World J Hepatol 2016;8:200-206. doi:10.4254/wjh.v8.i3.200

58. Williams K, Lewis JF, Davis G, Geiser EA. Dobutamine stress echocardiography in patients undergoing liver transplantation evaluation. Transplantation 2000;69:2354-2356. doi:10.1097/00007890200006150-00023

59. Rudzinski W, Waller AH, Prasad A, et al. New index for assessing the chronotropic response in patients with end-stage liver disease who are undergoing dobutamine stress echocardiography. Liver Transpl 2012;18:355-360. doi:10.1002/lt.22476

60. Oh JK, Kane GC. Diastolic stress echocardiography: the time has come for its integration into clinical practice. J Am Soc Echocardiogr 2014;27:1060-1063. doi:10.1016/j.echo.2014.08.013

61. Krag A, Bendtsen F, Dahl EK, Kjær A, Petersen CL, Møller S. Cardiac function in patients with early cirrhosis during maximal betaadrenergic drive: a dobutamine stress study. PLoS One 2014;9:e109179. doi:10.1371/journal.pone.0109179

62. Wong F, Girgrah N, Graba J, Allidina Y, Liu P, Blendis L. The cardiac response to exercise in cirrhosis. Gut 2001;49:268-275. doi:10.1136/ gut.49.2.268

63. Sampaio F, Lamata P, Bettencourt N, et al. Assessment of cardiovascular physiology using dobutamine stress cardiovascular magnetic resonance reveals impaired contractile reserve in patients with cirrhotic cardiomyopathy. J Cardiovasc Magn Reson 2015;17:61. doi:10.1186/ s12968-015-0157-6

64. Lancellotti P, Pellikka PA, Budts W, et al. The clinical use of stress echocardiography in nonischaemic heart disease: recommendations from the European Association of Cardiovascular Imaging and the American Society of Echocardiography. Eur Heart J Cardiovasc Imaging 2016;17:1191-1229. doi:10.1093/ehjci/jew190 
65. Pieske B, Tschöpe C, de Boer RA, et al. How to diagnose heart failure with preserved ejection fraction: The HFA-PEFF diagnostic algorithm: a consensus recommendation from the Heart Failure Association (HFA) of the European Society of Cardiology (ESC). Eur Heart J 2019;40:32973317. doi:10.1093/eurheartj/ehz641

66. Wiese S, Hove JD, Møller S. Cardiac imaging in patients with chronic liver disease. Clin Physiol Funct Imaging 2017;37:347-356. doi:10.1111/ cpf.12311

67. Lossnitzer D, Steen H, Zahn A, et al. Myocardial late gadolinium enhancement cardiovascular magnetic resonance in patients with cirrhosis. J Cardiovasc Magn Reson 2010;12:47. doi:10.1186/1532429X-12-47

68. Bernardi M, Calandra S, Colantoni A, et al. Q-T interval prolongation in cirrhosis: prevalence, relationship with severity, and aetiology of the disease and possible pathogenetic factors. Hepatology 1998;27:28-34. doi:10.1002/hep.510270106

69. Mozos I. Arrhythmia risk in liver cirrhosis. World J Hepatol 2015;7:662672. doi:10.4254/wjh.v7.i4.662

70. Pall A, Czifra A, Vitális Z, Papp M, Paragh G, Szabó Z. Pathophysiological and clinical approach to cirrhotic cardiomyopathy. J Gastrointestin Liver Dis 2014;23:301-310. doi:10.15403/jgld.2014.1121.233.apac

71. Trevisani F, Di Micoli A, Zambruni A, et al. QT interval prolongation by acute gastrointestinal bleeding in patients with cirrhosis. Liver Int 2012;32:1510-1515. doi:10.1111/j.1478-3231.2012.02847.x

72. Ge PS, Runyon BA. Role of plasma BNP in patients with ascites: Advantages and pitfalls. Hepatology 2014;59:751-753. doi:10.1002/ hep.26689

73. Campbell DJ, Mitchelhill KI, Schlicht SM, Booth RJ. Plasma aminoterminal pro-brain natriuretic peptide: A novel approach to the diagnosis of cardiac dysfunction. J Card Fail 2000; 6:130-139. doi:10.1016/S1071-9164(00)90015-0
74. Wong F, Siu S, Liu P, Blendis LM. Brain natriuretic peptide: is it a predictor of cardiomyopathy in cirrhosis? Clin Sci 2001;101:621628.

75. Ruiz-del-Árbol L, Serradilla R. Cirrhotic cardiomyopathy and circulatory function. World J Gastroenterol 2015;21:11502-11521. doi:10.3748/wjg.v21.i41.11502

76. Henriksen JH, Gotze JP, Fuglsang S, Christensen E, Bendtsen F, Moller S. Increased circulating pro-brain natriuretic peptide (proBNP) and brain natriuretic peptide (BNP) in patients with cirrhosis: relation to cardiovascular dysfunction and severity of disease. Gut 2003;52:15111517. doi:10.1136/gut.52.10.1511

77. Saner FH, Neumann T, Canbay A, et al. High brain-natriuretic peptide level predicts cirrhotic cardiomyopathy in liver transplant patients. Transpl Int 2011;24:425-432. doi:10.1111/j.1432-2277.2011.01219.x

78. Ponikowski P, Voors AA, Anker SD, et al; ESC Scientific Document Group. 2016 ESC Guidelines for the diagnosis and treatment of acute and chronic heart failure: The Task Force for the diagnosis and treatment of acute and chronic heart failure of the European Society of Cardiology (ESC) Developed with the special contribution of the Heart Failure Association (HFA) of the ESC. Eur Heart J 2016;37:2129-2200. doi:10.1093/eurheartj/ehw128

79. Ziada D, Gaber R, Kot N, Ghazy M, Nagy H. Predictive value of N-terminal pro B-type Natriuretic Peptide in Tissue Doppler-diagnosed cirrhotic cardiomyopathy. Heart Mirror J 2011;5:186-191.

80. Qureshi W, Mittal C, Ahmad U, et al. Clinical predictors of post-liver transplant new-onset heart failure. Liver Transpl 2013;19:701-710. doi:10.1002/1t.23654

81. Kimer N, Goetze JP, Bendtsen F, Moller S. New vasoactive peptides in cirrhosis: organ extraction and relation to the vasodilatory state. Eur Clin Invest 2014;44:441-452. doi:10.1111/eci.12249 